\title{
ESTUDIO DE LA INTERACCIÓN MAMÁ-BEBÉ PREMATURO A TRAVÉS DE LA ESCALA BRAZELTON Y ALGUNAS IMPLICACIONES SOBRE LA SALUD MENTAL DE LAS MADRES
}

\author{
Kelly Romero-Acosta ${ }^{1}$ \\ Corporación Universitaria del Caribe CECAR \\ César Argumedos De La Ossa ${ }^{2}$ \\ Universidade Federal de Mato Grosso (UFMT) \\ Diego Pérez Vásquez ${ }^{3}$ \\ Apsefacom - Valledupar
}

\section{Resumen}

La interacción madre-bebé es la acción que permite el vínculo entre la figura materna y su hijo, suscitando o no respuestas y/o retroalimentación entre ambos. Esta relación de interacción puede ser difícil, sobre todo si la madre presenta algún tipo de trastorno psicológico — tal como ansiedad, depresión, personalidad, entre otros-. Se ha encontrado que los bebés prematuros pueden nacer con una serie de complicaciones a nivel físico así, como también, de regulación de emociones. En la relación madre-bebé-prematuro, se ha demostrado que las madres de los infantes prematuros son más propensas a desarrollar, entre dos y tres veces, más dificultades psicológicas en comparación con las madres de los bebés nacidos a término. Con el fin de realizar la revisión bibliográfica, se revisaron

1 Doctora en Psicopatología de niños, adolescentes y adultos. Docente investigador tiempo completo. Programa de psicología. Corporación Universitaria del caribe-CECAR. Correo: kelly.romero@cecar.edu.co.orcid.org/0000-0002-6568-1316.

2 Psicólogo. Maestrando en Psicología. Universidade Federal de Mato Grosso (UFMT). Correo: cargumedod@cecar.edu.co.orcid.org/0000-0002-8664-2690.

3 Psicólogo. Especialista en Investigación aplicada a la Educación. Corporación Universitaria del Caribe-CECAR. Correo: diego.perez@cecar.edu.co. orcid.org/0000-0002-60890127. 
artículos científicos que estudian la interacción madre-infante en bebés prematuros a partir de la escala Brazelton. La escala Brazelton brinda valiosa información acerca de las competencias y las habilidades del bebé. A partir de esta revisión bibliográfica, sabemos que en Latinoamérica no hay estudios sobre la interacción madrebebé-prematuro, utilizando la escala Brazelton.

Palabras clave: bebé prematuro; interacción, salud mental, Brazelton; relaciones madre-hijo.

\section{Abstract}

The mother-baby interaction is the action that allows the link between the mother figure and her son. This interaction relationship can be difficult, especially in the mother. It has been found that premature babies can be born with a series of complications at a physical level as well as regulation of emotions. In the mother-baby-premature relationship, it has been shown that mothers of premature infants are more likely to develop two to three times more psychological compared to mothers of full-term babies. In order to carry out the literature review, the articles that are interested in the study of motherinfant interaction in premature babies from the Brazelton scaling are reviewed. Brazelton climbing provides valuable information about the skills and abilities of the baby. From this bibliographical review we know that in Latin America there are no studies on the motherbaby-premature interaction from the Brazelton escalation.

Keywords: premature infant; interaction, mental health, Brazelton; mother-child relationships. 


\section{Introducción}

La interacción puede definirse como una "acción que se ejerce recíprocamente entre dos o más objetos, agentes, fuerzas, funciones" - $\mathrm{y}$ que, en su aplicación a los seres humanos, hace referencia a las relaciones entre sí- (RAE, 2018). Ahora bien, en el contexto de la interacción madrebebé, el término se refiere a la acción entre madre-infante que suscita o no respuestas en ambas partes, y que a su vez se retroalimentan mutuamente; esta relación con las figuras cuidadoras se considera fundamental para la estructuración psíquica del niño (Leonardelli, 2009).

Durante los primeros meses de vida se despliega un proceso psicoafectivo y neuro-madurativo del niño, y las principales experiencias son cruciales para acomodarse al medio (Bowlby, 1995, 1998). Bowlby estudia la interacción madre-infante a través de la teoría del apego que es una de las conductas de interrelación más importantes, sucede entre la proximidad de un sujeto hacia otro, estableciéndose vínculos entre ellos (Bowlby, 1995). A partir del apego, el bebé puede mantener representaciones mentales de su madre, incluso, aunque ella no se encuentre presente; además, empieza a desarrollar una imagen de su cuidadora a partir de su interacción (Bowlby, 1982; Papalia \& Wendkos, 1992).

Para el estudio de la interacción madre-infante se pueden utilizar diferentes técnicas de observación, instrumentos y escalas que permiten conocer no solo el factor interactivo, sino también, el estado del bebé y de las variables que pueden afectar la relación materno-filial. Entre las escalas más usadas para la evaluación del recién nacido se destacan la Neonatal Behavioral Assesment Scale (NBAS) o Escala de Brazelton (Als, Butler, Kosta, \& McAnulty, 2005) y la Neurobehavioral Assessment of the Preterm Infant (NAPI) (Korner \& Thom 1990).

De acuerdo con varios autores, la NBAS es uno de los instrumentos de medición de mayor uso e interés para la evaluación neonatal. Esto es debido a que permite el registro de los efectos tempranos de la condición médica perinatal, en cuanto al comportamiento y al riesgo de los problemas posteriores al desarrollo. Además, tiene una importante aplicación clínica en la intervención para mejorar la vinculación madre-hijo en las muestras conformadas por infantes en riesgo (Brazelton, 1973a; Brazelton, 1973b; 
Estudio de la interacción mamá-bebé prematuro a través de la escala Brazelton y algunas implicaciones sobre la salud mental de las madres

Sostek \& Anders 1977; Holmes et al 1982; Green, Bax \& Tsitsikas, 1989; Brazelton \& Nugent 1995; Costa, et al., 2010).

La Escala Brazelton -NBAS-, cuya traducción en castellano es escala para la evaluación del comportamiento neonatal, permite ubicar situaciones de riesgo en el período neonatal, ofreciendo no solamente al clínico, sino también a los padres, la posibilidad de conocer mejor las capacidades y competencias del bebé. Esta escala fue desarrollada por el Dr. Brazelton, profesor emérito de la Harvard Medical School, y sus colegas, en 1973. Estos investigadores consideraron al recién nacido un ser activo y competente que viene al mundo dotado de ciertas características que le permiten comunicarse. El neonato es un ser dinámico que tiene la capacidad para proporcionar una respuesta a ciertos estímulos (Brazelton, 2001).

Algunas aplicaciones de la NBAS en el campo investigativo se han realizado teniendo en cuenta factores de riesgo tales como: la exposición a la cocaína (Richardson, Hamel, Golschmindt \& Day 1996; Phillips, Sharma, Premachandra, Vaughn y Reyes-Lee, 1996), la exposición al cigarrillo, la cafeína y/o el alcohol (Jacobson, Fein, Jacobson, Schwartz E Dowler, 1984). Para estos casos, la escala puede ofrecer la posibilidad de averiguar cómo se encuentran los mecanismos de reorganización del sistema nervioso central del bebé, el cual estuvo afectado por la exposición intrauterina de sustancias nocivas. También, se ha estudiado la influencia que tiene el NBAS en la maternidad, pues la escala se puede aplicar en presencia de los padres; ellos pueden cambiar su percepción del bebé conforme vean sus capacidades en el transcurso de la aplicación de la escala (Worobey \& Belsky, 1982). Otros estudios se han interesado por la revisión de las propiedades psicométricas del NBAS (Jacobson, Fein, Jacobson, \& Schwartz, 1984; Costa et al, 2010), por el análisis del comportamiento neonatal y su interacción en niños sin complicaciones (Green, Bax \& Tsitsikas, 1989; Honglin Zhu et al., 2007), por la prematuridad y por las enfermedades respiratorias (Myers, Jarvis, Creasey, Kerkering, 1992).

Ahora bien, como punto de partida, es importante mencionar que uno de los aspectos involucrados sobre la salud mental se relaciona con el componente psicológico; en este sentido, y en cuanto a la condición de nacimiento de los bebés prematuros, los padres experimentan una serie de tensiones y desbordamientos emocionales que pueden desencadenar síntomas ansiosos, depresivos, sentimientos de culpa, asimismo, de 
desorganización psíquica; todo ello por no completar de manera normal su etapa de gestación (González, 2009; 2010; Ruiz 2004). Otras de las implicaciones sobre la salud mental de las madres con hijos bajo esta condición es la presencia de síntomas relacionados con el trastorno de estrés postraumático, los cuales pueden generar un riesgo de padecimiento de hasta el doble para los primeros síntomas internalizantes mencionados e incluso hasta el triple, para los síntomas de estrés postraumático (PuigCalsina, et al., 2018; Bener, 2013).

\section{Generalidades de la Escala Brazelton}

La escala Brazelton es capaz de describir el estado de los sistemas autónomo, motor, estado de conciencia y atención social, en tanto nace de la necesidad de obtener un perfil sobre la organización funcional del neonato (Als, 1986). Estos sistemas los estudia de forma dimensional. También, evalúa cuatro dimensiones: fisiológica, motora, estado de conciencia y atención/interacción. La escala se preocupa por analizar la interrelación de estos sistemas, debido a que el neonato, para adaptarse al medio, debe integrar estas cuatro dimensiones. Por tal razón, la escala es más que una simple evaluación de presentación estímulo-respuesta. En su aplicación, el examinador desempeña un papel de observador-participante, en tanto, intenta facilitar las actuaciones y los recursos organizativos del pequeño. Sus ítems seriados permiten evaluar las cuatro dimensiones en el transcurso de la prueba, así, la estabilidad del sistema nervioso autónomo es posible evidenciarla por la presencia o ausencia de la labilidad del color de la piel, temblores y sobresaltos. El funcionamiento del sistema motor se evalúa con la observación del tono muscular, la madurez motora, el grado de actividad motora, y el nivel de integración de movimientos (manoboca, por ejemplo). La organización del estado — que es la capacidad del neonato de regular sus estados de conciencia_-, se valora por medio del nivel de irritabilidad, del momento de máxima excitación, la capacidad para sustraerse a estímulos negativos, la capacidad para ser consolado y calmarse, entre otros (Brazelton, 1973b).

La NBAS contiene 28 ítems conductuales que se valoran a partir de una escala de nueve puntos. Asimismo, contiene 18 ítems de reflejos que estiman el estado neurológico por medio de una graduación de cuatro puntos. En la segunda edición de la escala (Brazelton, 1984) se añadieron 7 
ítems, con el fin de evaluar el grado de fragilidad y la calidad de la conducta de niños de alto riesgo, los cuales evalúan la calidad de respuesta a partir de la cantidad de estímulos que necesita el neonato por parte del examinador para organizar las respuestas. Ahora bien, aunque en el manual de la prueba, no habla exactamente de su estandarización; existen estudios psicométricos de dicha escala con puntaciones típicas (Costas et al., 2010; Costas, Fornieles, Botet, Boatella, \& De Cáceres, 2007). La información sobre los ítems que se tienen en cuenta en la evaluación se presenta en la Tabla 1.

Tabla 1

Algunos ítems de la escala Brazelton

\begin{tabular}{lll}
\hline \multicolumn{1}{c}{ Ítems de comportamiento } & \multicolumn{1}{c}{ Ítems reflejos } & \multicolumn{1}{c}{$\begin{array}{c}\text { Ítems } \\
\text { suplementarios }\end{array}$} \\
\hline Disminución de la respuesta a & Prensión plantar & Calidad de la alerta \\
la luz & Babinski & $\begin{array}{l}\text { Esfuerzo para } \\
\text { mantener la }\end{array}$ \\
Disminución de la respuesta al & Clonus aquíleo & atención \\
sonajero & De búsqueda o de & Ayuda por parte del \\
Disminución de la respuesta a & puntos cardinales & examinador \\
la campanilla & Succión & Irritabilidad general \\
Orientación visual y auditiva & Glabela & Vigor y resistencia \\
inanimada & Movimientos pasivos- & Regulación del \\
Orientación visual y auditiva & brazos & estado \\
animada & Movimientos pasivos- & Resta \\
Alerta & Piernas & examinador \\
Tono general & Prensión palmar & \\
Madurez motora & Reflejo del escalón & \\
Rapidez de reacción & Enderezamiento & \\
Irritabilidad & Marcha automática & \\
Respuesta al abrazo & Reptación & \\
Capacidad de ser consolado & Incurvación del tronco & \\
Capacidad de consolarse & Desviación tónica de & \\
Habilidad mano-boca & cabeza y ojos & Nistagmo \\
Temblores & Reflejo tónico del cuello & \\
Sobresaltos & Reflejo de moro & \\
Labilidad del color de la piel & & \\
Sonrisas & & \\
\hline
\end{tabular}

Adicionalmente, la escala Brazelton cuenta con un módulo interactivo social, el cual busca, ante todo, que el neonato presente sus mejores competencias (Hawthorne, 2005). El examinador debe conocer las técnicas 
necesarias para lograr que el bebé consiga su mejor actuación (Brazelton $\&$ Nugent 1997). Algunos de estos neonatos solo pueden responder a una modalidad sensorial a la vez; por esta razón, solo se les presenta estímulos de una modalidad sensorial, con intensidad reducida, para obtener una mejor respuesta.

En efecto, el objetivo de la escala es obtener la mejor actuación del bebé, solamente con hecho de que el bebé demuestre ser capaz de responder al estímulo, así sea en el tercer intento, se considera superado el ítem evaluado. En cuanto a la evaluación sobre las capacidades de los niños frágiles (con el fin de que muestren sus capacidades) en ocasiones, es necesario ayudarles un poco. Por ejemplo, se les puede dar el chupete con la finalidad de suministrar un tipo de apoyo que les permita encontrar calma para responder a los estímulos lo mejor posible. Los ítems de regulación del estado pueden influir mucho en la interacción madre-hijo; por ejemplo, un niño que le cueste regular su estado y organizarlo podría no tener las herramientas para establecer una correcta interacción.

El ítem 32 de la escala evalúa irritabilidad general. La irritabilidad en la escala Brazelton se evalúa por el número de veces que el niño muestra irritabilidad, así como los estímulos que le han producido ese estado. El ítem de labilidad del estado valora el cambio del estado del bebé. La capacidad de ser consolado valora el número de maniobras que el examinador utiliza para calmar al recién nacido. Por el contrario, la capacidad de consolarse valora las estrategias que utiliza el niño para recuperar el estado de tranquilidad.

\section{Interacción madre-hijo}

Los seres humanos, debido a ciertos factores de vulnerabilidad, generan dependencia en los primeros momentos de su vida (Marrone, 2001). La interacción madre e hijo, es un intercambio constante para el desarrollo ontogenético, el cual puede influir en las habilidades y competencias de los niños (Pérez-López 2006). La sensibilidad de la interacción entre madre e hijo aparece por primera vez dentro de la teoría del apego (Ainsworth y colaboradores, 1978), especifican que la sensibilidad maternal es el intento de comunicación por medio de señales de las madres hacia los niños; pero esta definición se ha ido estructurando para la formulación de un amplio rango de conductas maternales que permitan una amplia aceptación en el mundo científico (Ainsworth, Blehar, Waters, \& Walls 1978). 
Desde una perspectiva diferente, la interacción materna es vista como un proceso eficiente, que implica un conjunto de señales e interacciones entre madre e hijo (Shin, Park, Ryu, \& Seomun, 2008; Ainsworth, Bell $\&$ Stayton 1974; Nicolaou, Rosewella \& Marlow, Glazebrook, 2009). Una madre emocionalmente sensible es capaz de conectarse con su hijo y de responder a las peticiones de este, formando así, un ambiente estructurado entre ambos. La sensibilidad, en la interacción madre e hijo, es apreciada como un indicador de estabilidad emocional, para el proceso de desarrollo del niño (Als, 1986; Fuertes, Lopes-Dos-Santos, Beehgly \& Tronick, 2009; Van Zeijl, et al., 2006).

Por otro lado, los problemas en la interacción madre-bebé pueden producirse por la presencia de ansiedad o depresión materna. La falta de comprensión, la inseguridad, la aversión a la mirada fija del infante por parte de la madre y el poco desarrollo neurobiológico, son algunos de los factores que pueden contribuir a la existencia de problemas en la interacción madre-bebé (Klein \& Feldman, 2007).

Field (1977) descartó que los problemas en la interacción madreinfante se deban directamente a la separación entre madre y bebé, debido al nacimiento prematuro. Este autor señala que los problemas en la interacción son debidos a que los bebés no cuentan con el suficiente desarrollo neurobiológico como para entablar una relación interactiva con la madre. Este desarrollo insuficiente puede suscitar en las madres algunas respuestas negativas, tales como: miedo, ansiedad, y/o depresión.

Algunos estudios han demostrado que los padres de los infantes prematuros son más propensos a desarrollar dificultades psicológicas, problemas en la interacción con el infante (en su relación madre-hijo), confusión en la interpretación de las señales del sus propios hijos, e incluso, se pueden generar dificultades que favorecen el desarrollo de consecuencias negativas en la relación de los padres (Als, Lester y Brazelton, 1979; Macey, Harmon \& Easterbrooks, 1987; Korja et al., 2008; Treyvaud et al., 2010).

Otros estudios revelan que los procesos de interacción madre-hijo durante el periodo del nacimiento pueden ser oportunos para influenciar de manera positiva la relación y familiarización de los miembros de la familia (De Chateau \& Wiberg, 1977; Smeriglio, 1981). La expresión afectiva materna como la "sonrisa", la "cara de juego" o la "expresión vocal animada" favorecen la expresión positiva y afectiva en el bebé (Brazelton, 
Tronick, Adamson, Als, \& Wise, 1975). Asimismo, otro de los aspectos importantes es la estimulación táctil; por ejemplo, se ha demostrado que al aplicarse en momentos de separación materna temprana tiene un efecto sobre la mejora en la reactividad al estrés, la organización del estado, la maduración fisiológica y la atención (Scadifi et al., 1990; Field, 1995; Wiggera \& Neumanna, 1999; Lehmann, Stohr, \& Feldon, 2000).

\section{Bebé prematuro}

De acuerdo con Amiel-Tison (1999a) los bebés pueden clasificarse en: bebé a término (de 37 a 42 semanas de gestación); bebé postérmino o posmaduro (después de 42 semanas de gestación) y bebé prematuro (de menos de 37 semanas de gestación). El bebé prematuro nace antes de la trigésima séptima semana de gestación (American Pregnancy Association, 2015) y tiene alto riesgo de padecer diversas alteraciones en su proceso de desarrollo (Institute of Medicine US, 2007). Al nacer antes de tiempo, tiene bajo peso -menos de 2.500 gramos- (Amiel-Tison, 1999b), y por ello, podría presentar problemas respiratorios (síndrome de dificultad respiratoria o SDR), piel delgada, casi traslúcida, rasgos de arrugas, cartílago del oído suave y flexible, vello corporal, llanto débil, clítoris agrandado o escroto pequeño, entre otros (Fava, Zorzi \& Bottos, 1992). Según Charpak et al. (2005) el bajo peso de los niños al nacer se debe, en muchos casos, a una alimentación regular de la madre.

De acuerdo con Reed \& Stanley (1977) algunos factores que influyen en el nacimiento de un bebé prematuro son la hipertensión materna, la infección urogenital y el embarazo múltiple; lo que puede ser causal de parto prematuro. En los infantes nacidos prematuramente se activa rápidamente el eje hipotalámico-hipofisario-adrenal (HPA) debido al estrés intenso al que se someten en la unidad de cuidados intensivos neonatal (Tu et al., 2007; Pignotti \& Donzelli, 2008).

Algunas de las características presentadas por los bebés prematuro a nivel físico: tamaño corporal más pequeño, musculatura y cerebro menos desarrollado (Holmes et al, 1982); menos sentido de alerta, menos receptividad a la estimulación, menor regulación de las emociones, más irritabilidad y desorganización (DiVitto y Goldberg, 1979; Korja et al. 2008; Feldman, 2009). Así mismo, los infantes prematuros son más vulnerables a los problemas emocionales y conductuales (en lo referido a 
exposición temprana al estrés), esta condición tiene un efecto negativo en las interacciones madre-hijo (Hayes \& Sharif, 2009; Treyvaud et al., 2010; Forcada-Guex, Pierrehumbert, Borghini, Moessinger, \& Muller-Nix, 2011; Habersaat et al., 2014).

La prematuridad, hasta hace algunas décadas, era una importante causa de muerte infantil. Sin embargo, en estos momentos los avances médicos, ayudan a que el riesgo disminuya considerablemente. Este proceso que tiene el bebé prematuro en su nacimiento puede alterar la interacción con el medio ambiente, en especial con la madre. A partir de todo lo anterior, nuestro objetivo es revisar los estudios sobre la interacción madre-neonato teniendo en cuenta la utilización de la escala Brazelton en bebés prematuros.

\section{Método}

Se realizó una revisión bibliográfica de los estudios publicados en las bases de datos PsycINFO, PsyArticles, Science Direct, Proquest, Francis \&Taylor y Scopus sobre la interacción madre-bebé prematuro usando la escala Brazelton. La búsqueda se realizó teniendo en cuenta las siguientes palabras claves: Brazelton, interaction y mother y se encontró 3.173 resultados en todas las bases de datos. La búsqueda se perfiló aún más al agregar un cuarto término clave: "premature birth", encontrando así un total de 1900 coincidencias. Solo se seleccionaron los artículos científicos con vínculo completo en inglés, los que incluyeran dentro de su investigación las variables a partir de las 4 palabras claves, y que además, estudiaran la relación madre-niño. Finalmente, realizado este filtro, se obtuvo como resultado un total de 7 artículos que estuvieron enfocados específicamente en estudio de las interacciones madre-infante en muestras de bebés prematuros a partir del uso de la Neonatal Behavioral Assesment Scale (NBAS). Por otro lado, se incluyó un artículo que no utilizó a la escala Brazelton, pero que se consideró útil para los objetivos de la revisión; uno de los autores de este artículo es el propio Brazelton. En la Tabla 2 se muestra un resumen de las investigaciones encontradas. 
Tabla 2

Artículos de la revisión bibliográfica

\begin{tabular}{|c|c|c|c|}
\hline $\begin{array}{c}\text { Autor (es), } \\
\text { año }\end{array}$ & Objetivo & Sujetos & Resultados \\
\hline Field, 1977. & $\begin{array}{l}\text { Investigar los } \\
\text { efectos de la } \\
\text { separación } \\
\text { temprana y } \\
\text { manipulaciones } \\
\text { experimentales } \\
\text { sobre las } \\
\text { interacciones cara a } \\
\text { cara madre-niño. }\end{array}$ & $\begin{array}{l}12 \text { prematuros } \\
\text { con dificultades } \\
\text { respiratorias } \\
\text { separados de } \\
\text { sus padres; } 12 \\
\text { post maduros } \\
\text { no separados; } \\
12 \text { a término. }\end{array}$ & $\begin{array}{l}\text { No se encontraron } \\
\text { diferencias significativas } \\
\text { entre la interacción de los } \\
12 \text { niños prematuros y los } \\
\text { doce niños postmaduros. } \\
\text { Esto le sugirió a la } \\
\text { autora que la separación } \\
\text { posiblemente no es un } \\
\text { causante de problemas en } \\
\text { la interacción. }\end{array}$ \\
\hline $\begin{array}{l}\text { Widmayer } \\
\& \text { Field, } \\
\text { (1981). }\end{array}$ & $\begin{array}{l}\text { Investigar sobre } \\
\text { la efectividad } \\
\text { del uso de la } \\
\text { escala Brazelton } \\
\text { para enseñar a } \\
\text { madres sobre el } \\
\text { comportamiento de } \\
\text { sus niños. }\end{array}$ & $\begin{array}{l}30 \text { niños } \\
\text { prematuros } \\
\text { sanos (nacidos } \\
\text { con menos de } \\
37 \text { semanas de } \\
\text { gestación). }\end{array}$ & $\begin{array}{l}\text { Los resultados de la } \\
\text { investigación indican } \\
\text { mejor respuesta en las } \\
\text { puntuaciones de los } \\
\text { grupos experimentales } \\
\text { (Brazelton/MABI y } \\
\text { MABI) en comparación } \\
\text { al grupo control en los } \\
\text { componentes de los } \\
\text { procesos interactivos, de } \\
\text { alimentación y secuencia } \\
\text { cara a cara a partir del uso } \\
\text { de la escala Brazelton y su } \\
\text { adaptación. }\end{array}$ \\
\hline
\end{tabular}




\begin{tabular}{|c|c|c|c|}
\hline $\begin{array}{c}\text { Autor (es), } \\
\text { año }\end{array}$ & Objetivo & Sujetos & Resultados \\
\hline $\begin{array}{l}\text { Lester, } \\
\text { Hoffman, } \\
\text { Joel, } \\
\text { Brazelton, } \\
1985 .\end{array}$ & $\begin{array}{l}\text { Examinar el } \\
\text { ritmo social de } \\
\text { interacción madre- } \\
\text { niño. }\end{array}$ & & $\begin{array}{l}\text { Existencia de } \\
\text { comportamientos } \\
\text { periódicos en la diada } \\
\text { madre- niño y que } \\
\text { además proporcionan una } \\
\text { estructura temporal para } \\
\text { la organización cognitivo- } \\
\text { afectiva. Las diferencias en } \\
\text { la sincronía entre los bebés } \\
\text { a término y los prematuros } \\
\text { pueden explicarse } \\
\text { luego del informe de las } \\
\text { diferencias del lenguaje } \\
\text { entre estos grupos. }\end{array}$ \\
\hline $\begin{array}{l}\text { Feldman, \& } \\
\text { Eidelman, } \\
2003 .\end{array}$ & $\begin{array}{l}\text { Examinar los } \\
\text { efectos de la leche } \\
\text { materna sobre el } \\
\text { desarrollo de los } \\
\text { infantes. }\end{array}$ & $\begin{array}{l}87 \text { niños } \\
\text { prematuros. }\end{array}$ & $\begin{array}{l}\text { A mayor cantidad de leche } \\
\text { materna, mejor desarrollo } \\
\text { neuro-comportamental, } \\
\text { mayor alerta durante las } \\
\text { interacciones sociales y } \\
\text { mayor contacto táctil por } \\
\text { parte de las madres. A } \\
\text { mayores puntuaciones en } \\
\text { depresión materna más } \\
\text { bajas cantidades de leche } \\
\text { materna, bajo contacto } \\
\text { corporal madre- bebé y } \\
\text { más bajas competencias } \\
\text { cognitivas }\end{array}$ \\
\hline $\begin{array}{l}\text { Meier, et al., } \\
2003 .\end{array}$ & $\begin{array}{l}\text { Investigar la } \\
\text { relación entre } \\
\text { sensibilidad } \\
\text { materna e } \\
\text { irritabilidad del } \\
\text { infante. }\end{array}$ & $\begin{array}{l}29 \text { niños } \\
\text { prematuros. }\end{array}$ & $\begin{array}{l}\text { En el primer mes neonatal } \\
\text { no hubo influencia de } \\
\text { la sensibilidad materna } \\
\text { sobre el llanto del bebé. } \\
\text { Asimismo, la irritabilidad } \\
\text { puede ocurrir a pesar } \\
\text { de una alta sensibilidad } \\
\text { materna, tal y como sucede } \\
\text { en los niños a término. }\end{array}$ \\
\hline
\end{tabular}




\begin{tabular}{|c|c|c|c|}
\hline $\begin{array}{c}\text { Autor (es) } \\
\text { año }\end{array}$ & Objetivo & Sujetos & Resultados \\
\hline $\begin{array}{l}\text { Feldman \&. } \\
\text { Eidelman, } \\
2006\end{array}$ & $\begin{array}{l}\text { Examinar las } \\
\text { relaciones entre la } \\
\text { neuromaturación, } \\
\text { interacción madre- } \\
\text { hijo y el desarrollo } \\
\text { cognitivo en } \\
\text { recién nacidos } \\
\text { prematuros. }\end{array}$ & $\begin{array}{l}120 \text { neonatos } \\
\text { prematuros } \\
\text { nacidos } \\
\text { con <36 de } \\
\text { gestación. }\end{array}$ & $\begin{array}{l}\text { Las madres de los infantes } \\
\text { con un peso al nacer < } 10 \\
\text { percentil reportan ser más } \\
\text { intrusivas en la relación } \\
\text { de interacción madres- } \\
\text { hijo, así como también } \\
\text { se evidencia un mayor } \\
\text { compromiso negativo en } \\
\text { comparación con los otros } \\
\text { grupos de estudio de la } \\
\text { investigación. }\end{array}$ \\
\hline $\begin{array}{l}\text { Habersaat et } \\
\text { al, } 2014 .\end{array}$ & $\begin{array}{l}\text { Evaluar el impacto } \\
\text { de la intervención } \\
\text { temprana en la } \\
\text { relación madre-hijo } \\
\text { en recién nacidos } \\
\text { prematuros. }\end{array}$ & $\begin{array}{l}60 \text { niños } \\
\text { nacidos en } \\
\text { menos de } 33 \\
\text { semanas de } \\
\text { gestación, entre } \\
2005 \text { y 2009, y } \\
\text { hospitalizados } \\
\text { en la UCIN } \\
\text { del Hospital } \\
\text { Universitario de } \\
\text { Lausana. }\end{array}$ & $\begin{array}{l}\text { Se encontró una } \\
\text { diferencia significativa } \\
\text { en la comparación de } \\
\text { grupo en los niveles de } \\
\text { cortisol a los } 6 \text { meses, } \\
\text { mostrándose más bajos en } \\
\text { los niños prematuros sin } \\
\text { intervención. Sin embargo, } \\
\text { en la medición de los } 12 \\
\text { meses, no se encontró } \\
\text { diferencias entre los } \\
\text { grupos. }\end{array}$ \\
\hline
\end{tabular}


Estudio de la interacción mamá-bebé prematuro a través de la escala Brazelton y algunas implicaciones sobre la salud mental de las madres

\begin{tabular}{|c|c|c|c|}
\hline $\begin{array}{c}\text { Autor (es), } \\
\text { año }\end{array}$ & Objetivo & Sujetos & Resultados \\
\hline $\begin{array}{l}\text { Holditch- } \\
\text { Davis et al } \\
2014 \text {. }\end{array}$ & $\begin{array}{l}\text { Examinar las } \\
\text { intervenciones } \\
\text { administradas } \\
\text { por vía materna } \\
\text { en recién nacidos } \\
\text { prematuros } \\
\text { sobre la angustia } \\
\text { psicológica } \\
\text { materna, estrés } \\
\text { de crianza, y la } \\
\text { relación madre- } \\
\text { infante. }\end{array}$ & $\begin{array}{l}240 \text { neonatos } \\
\text { (con sus } \\
\text { madres) } \\
\text { pertenecientes a } \\
4 \text { hospitales de } \\
\text { EE. UU }\end{array}$ & $\begin{array}{l}\text { Los efectos de mostraron } \\
\text { un mejor resultado en el } \\
\text { grupo con intervención } \\
\text { en la interacción madres- } \\
\text { infante encontrando } \\
\text { un efecto menor sobre } \\
\text { el estrés en la crianza. } \\
\text { Así mismo, se reportó } \\
\text { beneficios en la capacidad } \\
\text { de respuesta de las } \\
\text { interacciones, en el peso } \\
\text { de los bebés, en el sueño, } \\
\text { llanto, regulación de la } \\
\text { respiración y crecimiento } \\
\text { de la cabeza. En general, } \\
\text { se encontraron efectos } \\
\text { positivos sobre la angustia } \\
\text { psicológica materna y } \\
\text { las interacciones (sobre } \\
\text { todo a corto plazo), } \\
\text { sin embargo, se deben } \\
\text { seguir estudiando, } \\
\text { con el fin de seguir } \\
\text { examinando la eficacia } \\
\text { de las intervenciones a } \\
\text { largo plazo y sobre si } \\
\text { alguna técnica es mejor } \\
\text { que la otra, o si se deben } \\
\text { combinar. }\end{array}$ \\
\hline
\end{tabular}




\section{Resultados}

A continuación, se presentan los resultados, las generalidades y características de la interacción madre-bebé prematuro; esto se hizo dividiendo los resultados en varios subpuntos.

\section{Generalidades en la interacción madre-bebé-prematuro}

Como fue señalado anteriormente, la interacción madre e hijo se caracteriza por tener un intercambio constante en la comunicación de la diada, y la calidad de este intercambio puede influir en las habilidades comunicativas de los mismos (Pérez-López 2006). Esa comunicación constante tiene un ritmo determinado. En la búsqueda bibliográfica se halló un interesante trabajo sobre el ritmo en la interacción de la madre y del bebé prematuro y a término. Lester et al. (1985) cuantificaron los ritmos de interacción social en bebés prematuros y a término (de tres a cinco meses de edad).

Los autores mencionados se basaron en la existencia de "ciclos" biológicos en los seres humanos, tales como el ciclo del sueño, el ritmo circadiano, el ritmo cardiaco, entre otros. A partir de ello, se pensó que la interacción madre-hijo tiene un ritmo, basado en miradas, sonidos y movimientos. Para estudiar la interacción se filmaron cara a cara las diadas durante tres minutos. Los resultados mostraron la existencia de periodicidad en los comportamientos de cada infante y de sus madres (entre 0,22 y, 10Hz). También se encontró un incremento de periodicidad entre los tres y cinco meses, los bebés a término mostraron más coherencia que los prematuros en su periodicidad y frecuentemente, ellos mismos iniciaban la interacción llamando la atención de la madre, los bebés prematuros presentaron más dificultades en iniciar la interacción con su madre. Lester et al. (1985) indican que los ciclos de atención e inatención se reconocen como parte del desarrollo de la interacción social y, por supuesto, estos ciclos en la interacción madre-hijo pueden ser cruciales para las experiencias afectivas y para la organización cognitiva del neonato. Como se puede apreciar, en los bebés prematuros este ritmo puede verse afectado en periodicidad y frecuencia, entonces la interacción puede verse afectada dependiendo de las habilidades del propio bebé. 
Sabemos que la interacción madre-bebé puede verse influida por las habilidades del bebé prematuro. En estudios anteriores se ha encontrado que los neonatos prematuros tienen dificultades para mantener la atención visual durante el juego (Eckerman, Hsu, Molitor Leung \& Goldstein, 1999) y no pueden autorregularse tan bien como un bebé a término. Estos factores, combinados con el decremento de la sensibilidad materna, producen una baja sincronía entre las madres y sus bebés (Lester et al., 1985).

Vale la pena preguntarse: ¿tendrá la sensibilidad materna relación con la irritabilidad del bebé prematuro en la interacción madre-bebé? Meier, Wolke, Gutbrod \& Rust (2003) estudiaron la relación entre irritabilidad y sensibilidad materna en 29 niños prematuros y sus madres. Ellos definieron como "sensiblidad" a la capacidad materna para reconocer, interpretar y responder apropiadamente a la comunicación y a las necesidades del bebé.

Para llevar a cabo el estudio se utilizaron tres escalas: la escala Brazelton, la escala madre-bebé-Mother and Baby Scale, MABS- (James-Robert, 1988) y la Crying Pattern Questionaire-CPQ (James-Roberts, \& Halil, 1991); también, se realizó una entrevista filmada, semi-estructurada, de 6 minutos de duración (2,5 min. juego; 2,5 min. juego libre; 1 min. observando al bebé ver a su madre). Se encontró que en el primer mes no hubo influencia alguna de la sensibilidad materna sobre el llanto del bebé. Así mismo, la irritabilidad pudo ocurrir a pesar de una alta sensibilidad materna, tal y como sucede en los niños a término. Entonces, la irritabilidad que puede presentar un bebé prematuro se da independientemente de la capacidad de las madres para reconocer, interpretar y responder a las necesidades del bebé. Sin embargo, aunque la sensibilidad materna no se relacione con la irritabilidad del bebé, influiría en la interacción madre-bebé propiamente dicha. Ciertamente una madre que no sea capaz de reconocer, interpretar y responder adecuadamente a los llamados del bebé no podría mantener una frecuencia y una periodicidad adecuadas del ritmo de la interacción. No obstante, se requieren más estudios al respecto.

\section{Influencia de la madre en la interacción madre-bebé}

La interacción cara a cara proporciona una base para el desarrollo de las herramientas comunicativas del bebé. El interés del bebé por la cara de la madre se despierta antes del interés por los objetos, por tanto, puede considerarse como básico para la posterior adaptación al medio. En un 
estudio realizado por Field, en 1977, se formaron tres grupos: (a) se le aplicó la escala Brazelton a 12 bebés separados de sus madres, prematuros y con estrés respiratorio; (b) se les aplicó la escala Brazelton a 12 bebés con sus madres presentes, y postmaduros; y (c) se les aplicó la escala Brazelton a 12 bebés sanos y a término, no separados de sus madres.

Los resultados del instrumento en los dos primeros grupos fueron bajos, tanto en los ítems de orientación animada e inanimada, como en los ítems de consolación y respuesta al abrazo. En las puntuaciones motoras, las cuales incluyeron interacción cara a cara, madurez muscular, tono muscular y flexibilidad motora, también puntuaron bajo. No se encontraron diferencias significativas entre la interacción de los posmaduros y prematuros. La autora sugiere que la separación contribuye menos sobre los problemas de interacción madre-bebé, que los déficits tempranos encontrados en la escala Brazelton. En general, se pudo observar que hubo diferencias entre la interacción de los bebés normales y los de alto riesgo. Si los bebés solo hubiesen sido observados, se atribuiría a un efecto de la separación temprana, sin embargo, esta información se cotejó con los resultados de la escala Brazelton y en los resultados fue más significativo el déficit temprano que la separación temprana.

También se halló en un estudio muy interesante el efecto que la intervención de las madres tenía sobre el bebé (Holditch-Davis et al., 2014). Se trataban de bebés prematuros que necesitaban intervenciones administradas por las madres. Se examinó el efecto de estas intervenciones sobre la angustia psicológica, materna síntomas depresivos, ansiedad, estrés postraumático - EPT - y al igual que el estrés de crianza) y la relación madre-infante. El estudio estuvo conformado por 240 madres organizadas de forma aleatoria en tres grupos: un para la aplicación de la técnica de intervención auditivo-táctil-vestibular visual-vestibular (ATVV); otro para la técnica de atención canguro (KC) y el último para realizar una atención del grupo control.

También se halló en un estudio muy interesante el efecto que la intervención de las madres tenía sobre el bebé (Holditch-Davis et al., 2014). Se trataba de bebés prematuros que necesitaban intervenciones administradas por las madres. Se examinó el efecto de estas intervenciones sobre la angustia psicológica materna, síntomas depresivos, ansiedad, estrés postraumático -TEPT- estrés hacia la crianza y la relación madre-infante. 
Estudio de la interacción mamá-bebé prematuro a través de la escala Brazelton y algunas implicaciones sobre la salud mental de las madres

El estudio estuvo conformado por 240 madres organizadas en tres grupos, dos grupos recibieron intervención y uno no.

Se utilizaron instrumentos de cribado para depresión, ansiedad y estrés postraumático. También, se aplicaron escalas que medían estrés de la crianza, preocupación por la salud infantil, vulnerabilidad infantil y la escala Brazelton -esta última para medir la capacidad de respuesta de los infantes en el alta hospitalaria.Se halló que el estrés en la crianza de los hijos fue menor para las madres que recibieron intervención. Del mismo modo, se encontró que, dentro de los efectos, las intervenciones tienen importantes beneficios para los lactantes como: un mejor aumento de peso, aumento de la lucidez mental (antes y después de las comidas), una mayor capacidad de respuesta durante las interacciones madre-infante, más sueño, menos llanto, mayor regularidad respiratoria y mejor crecimiento de la cabeza en comparación con el grupo control. Sin embargo, aunque los resultados tuvieron efectos positivos sobre la angustia psicológica materna y las interacciones, su eficacia pudo ser comprobada en mejor medida en un periodo de corto plazo, por lo que requiere futuros estudios que permitan comprobar su efecto a largo plazo y el uso continuo de las intervenciones. De igual manera, en cuanto a otros resultados, se encontró que uno de los dos grupos de intervención fue más alto que el otro, por lo que no es posible aseverar si estas intervenciones pueden combinarse de manera efectiva.

\section{Influencia del desarrollo neuromotor y cognitivo en la interacción madre-bebé prematuro}

Ya sabemos que la madre influye en la buena interacción con su bebé prematuro, ahora bien, ¿el desarrollo del bebé cómo puede afectar esa relación? Feldman \& Eidelman, en el 2006, examinaron la relación entre la maduración neurológica del bebé, su desarrollo cognitivo y la relación madre-hijo. Participaron neonatos prematuros, nacidos entre las 25 y 35 semanas (o <36 semanas) de gestación y con un peso entre los 530 y 1790 gramos. Para la evaluación de la interacción madre-bebé prematuro se utilizó la escala Brazelton a los 3 y 24 meses.

Se encontró que las madres cuyos bebés se encontraban más inmaduros, mostraron un comportamiento significativamente más intrusivo 
a los tres y a los veinticuatro meses, en comparación con los otros grupos de madres.

\section{Otros aspectos que influyen en la interacción madre-bebé}

\section{Efectos positivos de la aplicación de la escala Brazelton en la interacción madre-bebé}

A partir de esta revisión bibliográfica conocemos que la observación y evaluación de bebés utilizando la escala Brazelton puede tener efectos positivos en la percepción de la madre sobre las habilidades de su hijo. En la década de los ochenta, Widmayer \& Field (1981) realizaron un estudio sobre el efecto de la escala Brazelton en las madres, a ellos les interesaba observar si enseñar a las madres sobre el comportamiento de sus infantes tenía un efecto positivo en ellas. La muestra estuvo conformada por 30 niños neonatos prematuros sanos (<37 semanas de gestación) hijos de madres afroamericanas y con bajo nivel socioeconómico. Los bebés prematuros fueron asignados aleatoriamente a uno de tres grupos (dos experimentales y uno control). Se realizaron cuatro mediciones en cada grupo: al mes, a los cuatro meses y a los doce meses. En el primer grupo experimental las madres estuvieron presentes en la aplicación de la escala Brazelton y luego se les pidió que respondieran una serie de preguntas sobre el comportamiento de su recién nacido; en el segundo grupo experimental las madres no observaron la aplicación de la escala Brazelton, pero se les solicitó que respondieran las preguntas sobre el comportamiento de su recién nacido; Al tercer grupo, llamado control, no se le aplicó la escala Brazelton, pero si completaron un cuestionario sobre los hitos del desarrollo de sus bebés.

Las comparaciones de los grupos durante el mes inicial indicaron que el primer y segundo grupo experimental mostraron mejores puntuaciones en los procesos interactivos (como el cara a cara) y de alimentación en comparación con el grupo control. En el cuarto mes la interacción caraa-cara de los dos grupos experimentales fue significativamente mejor que el grupo control. Finalmente, en el décimo segundo mes los bebés de los grupos experimentales presentaron mejores puntuaciones en una escala sobre desarrollo mental, en comparación con el grupo control.

Los autores concluyen que compartir con las madres las habilidades del bebé prematuro a partir de la escala Brazelton puede mejorar la 
Estudio de la interacción mamá-bebé prematuro a través de la escala Brazelton y algunas implicaciones sobre la salud mental de las madres

interacción de la madre y del bebé, y con ello se puede contribuir a la mejora de su desarrollo cognitivo.

\section{Efectos de la leche maternal en la interacción madre- bebé prematuro}

Feldman \& Eidelman (2003) se interesaron por examinar los efectos de la leche materna (LM) sobre el desarrollo del bebé prematuro. Para ello, organizaron tres grupos: 1) recepción mínima ( $<25 \%$ de nutrición), recepción media (25\%-74\%) y recepción substancial de leche materna (> 75\%). Para llevar a cabo el estudio se analizó un video y se aplicó la escala Brazelton, con el fin de conocer la madurez neurocomportamental. Se argumentó en la investigación que la LM, debido a su contenido nutricional, es considerada como un factor que tiene un impacto directo sobre el funcionamiento cognitivo y neuronal del bebé, en especial de los prematuros.

Para obtener los resultados se explicitaron muy claramente las categorías a tener en cuenta en la videograbación, a saber, mirada fija materna; afecto maternal; habla maternal; contacto maternal; y estado del infante. Así mismo, se aplicó a las madres el Beck Depression Inventory —BDI- (Beck 1978) y a los niños la escala Brazelton. Se les aplicó aproximadamente de 36 a 38 semanas, es decir, cuando se les daba de alta.

Se encontró que las madres quienes proporcionaban una cantidad substancial de leche establecían significativamente más contacto táctilafectivo que las madres pertenecientes a los otros dos grupos. No obstante, las madres que no daban una cantidad significativa de leche materna hablaban mucho más con los niños que las otras madres. Se halló diferencias significativas entre los neonatos que recibieron altas cantidades de leche materna y los otros dos grupos; este grupo de neonato obtuvo los mejores puntajes en la escala Brazelton. Por otra parte, se halló alta correlación entre la depresión materna y el contacto afectivo táctil. No hubo correlación entre la depresión materna y funcionamiento neuro-comportamental. En la Tabla 3 se encuentra la significancia de la leche materna. La investigación comprobó que la ingestión de altas cantidades de leche materna en el periodo neonatal de niños prematuros se relaciona con un elevado peso y un mejor desarrollo neurológico y cognitivo. 


\section{El impacto de la intervención de la relación madre- bebé-prematuro}

En nuestra revisión bibliográfica encontramos un artículo que utilizó la escala Brazelton para medir el impacto de la intervención temprana en la interacción madre-hijo en bebés prematuros (Habersaat et al, 2014). La muestra estuvo constituida por 60 niños recién nacidos prematuros hospitalizados, quienes se dividieron en dos grupos: (a) bebés prematuros con intervención; (b) bebés prematuros sin intervención; y un grupo control de bebés nacidos a término. El programa de intervención se estructuró en tres fases; la primera: 33 semanas después de la concepción del bebé; la segunda a las 42 semanas; y la tercera a los 4 meses.

La primera fase de la investigación consistió en una observación conjunta de las madres y sus bebés en un procedimiento de atención estándar en la Unidad de Cuidados intensivos; en este procedimiento se exponía al bebé a ciertos estímulos para ver su desarrollo; estas observaciones tuvieron una duración aproximada de 60 y 70 minutos y se grabaron en videos.

En la segunda fase se utilizó la escala de evaluación del Comportamiento Neonatal de Brazelton para la identificación de las capacidades de los bebés; de igual modo, el proceso se registró en video.

En la tercera fase se realizó una guía de interacción estructurada programada en tres citas durante tres semanas consecutivas (McDonough, 2005); en cada cita se le pedía a las madres que jugaran libremente con su bebé durante 10 minutos. Todas las interacciones fueron grabadas en video y las madres estaban acompañadas por un especialista de orientación interactiva; el objetivo era promover las cualidades de cuidado y mejorar la sensibilidad de los padres hacia el bebé a través de la observación; estas citas tuvieron una duración entre 60 y 90 minutos en promedio.

Posteriormente, se convocó a los dos grupos casos y al grupo control para realizar la misma actividad de juego y registrarla nuevamente en video. Los niveles de cortisol diurno salival se evaluaron a los 6 y 12 meses. Los resultados de la investigación reportaron un impacto significativo de la intervención en los niveles de cortisol diurno a los 6 meses, los cuales fueron más bajos en los recién nacidos prematuros sin intervención en comparación con otros grupos. A los 12 meses, no se encontró tal diferencia. 
Estudio de la interacción mamá-bebé prematuro a través de la escala Brazelton y algunas implicaciones sobre la salud mental de las madres

\section{Discusión}

El fenómeno de la interacción madre-bebé ha sido estudiado de múltiples maneras y teniendo en cuenta diferentes situaciones. Ciertamente, los bebés no pueden expresar sus primeras palabras hasta casi un año después de nacer, sin embargo, pueden interactuar con el mundo que les rodea a partir de un rico vocabulario corporal, gestual, visual y, sobre todo, a través del llanto. Por esta razón, la escala Brazelton tiene en cuenta un amplio rango de comportamientos del bebé, permitiendo obtener un perfil del infante a partir del conocimiento de sus fortalezas, respuestas adaptativas y posibles puntos vulnerables. Todas estas características han facilitado que sea mayormente aplicada en el área clínica y de la investigación, tanto en neonatos sanos, como en aquellos que se encuentran en riesgo (Brazelton $\&$ Nugent, 1997).

En las investigaciones revisadas se estudió la interacción madreneonato a partir del fenómeno "cara a cara" y de la comparación de resultados con la escala Brazelton. Se sabe que algunos de los problemas de interacción madre-bebé pueden deberse a factores tales como: la presencia de ansiedad o depresión materna, la aversión de la madre a la mirada fija del infante, el poco desarrollo neurobiológico (mucho más si es prematuro) y/o a la sobre-estimulación materna (Belsky \& Fearon, 2002). Sumado a esto, se encuentran otros factores importantes: el bajo nivel socioeconómico, los problemas maritales y la presencia de psicopatologías en la madre (Zeanah, Boris \& Larrieu, 1997).

Ahora bien, iniciando con la investigación de Field (1977) a partir de la escala Brazelton, se ha descartado que la separación madre-niño tuviera un efecto directo y altamente significativo en la interacción de ambos. De acuerdo con este autor, la interacción madre-infante puede verse más perturbada por el poco desarrollo neurobiológico del neonato, por la baja madurez motora, por el bajo tono muscular y por las bajas puntuaciones en consolación y orientación inanimada y animada, que por la separación madre-bebé propiamente dicha. En efecto, estos déficits contribuyen a que la conducta de los recién nacidos de alto riesgo afecte la interacción madrebebé (Brazelton \& Nugent, 1995).

Cuando el bebé nace a término y en condiciones normales, puede establecer el control de su conducta y organizar sus funciones fisiológicas. 
Por el contrario, los prematuros, tienen que completar el desarrollo de estas habilidades fuera del vientre de la madre. También es cierto que cuando un bebé presenta cualquier dificultad, la respuesta de los padres hacia el niño puede estar ausente de tranquilidad y aceptación inmediatas, esto influiría negativamente en la estabilidad de la diada materno-filial, sobre todo si la madre se ve superada por la situación.

Ciertamente, las condiciones psicológicas de la madre pueden facilitar las condiciones de adaptación del bebé prematuro. En efecto, existe evidencia empírica que avala la importancia que tiene la relación madre-hijo, en el desarrollo, mantenimiento y cura de síntomas de carácter psicológico, especialmente en la infancia temprana, esto es, cuando ese bebé prematuro ya ha superado el año de vida (Chu \& Lieberman, 2010; Gunnar \& Quevedo, 2007; Romero-Acosta \& Ruiz, 2015).

Esta realidad se acerca a los hallazgos de la mayor parte de los estudios revisados en la presente investigación, por ejemplo, en Habersaat et al. (2014) ha informado que la vivencia del estrés es mayor en los niños prematuros en comparación con sujetos en su misma condición que fueron intervenidos para mejorar la eficacia de la relación madre-hijo. Estos hallazgos tienen relación con otros estudios que han encontrado que la interacción establecida por la madre sobre el lactante-prematuro tiene un papel importante sobre la autorregulación del estrés y sobre el apoyo de las experiencias difíciles que experimenta el infante (Feldman, 2007; Warren et al, 2003; Ahnert, Gunnar, Lamb, \& Barthel, 2004).

En línea con estos hallazgos, Widmayer \& Field (1981) encontraron que existe una mejor interacción madre-bebé cuando se utiliza la escala Brazelton en presencia de las madres. De manera similar Holditch et al. (2014), Feldman \& Eidelman (2006) y otros (White-Traut, Schwertz, McFarlin, \& Kogan, 2009; Ahn, Lee \& Shin, 2010; Neu \& Robinson, 2010) hallaron que las interacciones materno-filiales pueden mejorar con intervención y que esto a su vez optimiza la respuesta al estrés de los niños prematuros, sobre todo en la regulación del llanto, en el crecimiento y en la respiración.

Es necesario destacar que la escala Brazelton es cualitativa, da un perfil general sobre el estado del neonato y puede ayudar a los padres a valorar mucho más las potencialidades del bebé. En ese caso, el solo hecho de aplicarla junto a la madre podría influir en una mejoría de la relación 
madre-niño. A partir de esta revisión bibliográfica damos cuenta sobre el uso de la escala Brazelton dentro del campo investigativo en la relación madre-infante prematuro, presentando en nuestros resultados los aportes de los estudios encontrados.

Estos hallazgos pueden contribuir al desarrollo de nuevas líneas de investigación; por ejemplo, en Colombia no se encontraron estudios que indaguen sobre la interacción madre e hijo en bebés prematuros, y mucho menos que apliquen la escala Brazelton en bebés.

Debido a nuestra realidad y a la alta prevalencia de adolescentes embarazadas en el país (De cero a siempre, 2013), se hace necesario una ruta investigativa que permita estudiar la interacción madre-bebés prematuros en las jóvenes mamás colombianas, pues en las adolescentes se encuentran cifras alarmantes de nacimientos prematuros; esto puede terminar afectando la salud mental de las madres en su proceso de interacción con los hijos (Redacción el país, 2014; Opinión \& Salud 2015). Con el uso de la escala Brazelton como instrumento de medición y de soporte se pueden proponer nuevas investigaciones que aporten a la discusión teórico-científica, asimismo, que contribuyan al campo de la salud pública a nivel nacional.

Ciertamente, a partir de esta revisión teórica, sabemos que la escala Brazelton es un instrumento fiable y útil para ser utilizado en neonatos, sobre todo en prematuros. De acuerdo con los estudios revisados y disponibles sobre el tema, se recomienda que se realicen más investigaciones de corte longitudinal, con el fin de comprender mejor la interacción madre-hijo, y monitorear así los aspectos psicológicos que pudieran afectar la salud mental de las madres cuyos bebés han tenido un nacimiento prematuro, de esta manera se podrían crear estrategias de intervención psico-emocionales que aborden esta problemática. 


\section{Referencias}

Ahn, H. Y., Lee, J., \& Shin, H. J. (2010). Kangaroo care on premature infant growth and maternal attachment and post-partum depression in South Korea. Journal of Tropical Pediatrics, 56, (5), 342-344. http://dx.doi.org/10.1093/tropej/fmq063.

Ahnert, L., Gunnar, M. R., Lamb, M. E., \& Barthel, M. (2004). Transition to child care: Associations with infant-mother attachment, infant negativeemotion, and cortisol elevations. Child Development, 75 (3), 639-650. doi:10.1111/j.1467-8624.2004.00698.x.

Ainsworth M, Bell S, \& Stayton D. (1974). Infant/mother attachment and social development: "Socialization" as a product of reciprocal responsiveness to signals. En P. M. Richards (Ed.), Integration of a child into a social world; (pp. 99-135). Cambridge, UK: Cambridge University Press.

Ainsworth, M.D., Blehar, M., Waters, E., \& Wall, S. (1978). Patterns of attachment: A psychological study of the strange situation. Hillside, NJ. Lawrence Erlbaum Associates.

Als H. A (1986). Synactive model of neonatal behavioural organization: Framework for the assessment of neurobehavioural development in the premature infant and for support of infants and parents in the neonatal intensive care environment. Physical Occupational Therapy Paediatrics. 6 (3), 3-53. https://doi.org/10.1080/J006v06n03_02.

Als, H., Butler, S., Kosta, S. \& McAnulty, G. (2005), The Assessment of Preterm Infants' Behavior (APIB): Furthering the understanding and measurement of neurodevelopmental competence in preterm and full-term infants. Developmental. Disabilities Research. Reviews., 11 (1), 94-102. Doi:10.1002/mrdd.20053.

Als, H., Lester, B. M., \& Brazelton, T. B. (1979). Dynamics of behavioral organization of the premature infant: A theoretical perspective. In T. M. Field, A. M. Sostek, S. Goldberg, \& H. H. Sherman (Eds.), Infants bom at risk (pp. 173-192). New York: Spectrum Books.

American Pregnancy Asosiation (2015). Premature Birth Complications. Risks of complications for premature newborns. Retrieved from http://americanpregnancy.org/labor-and-birth/premature-birth-complications/. 
Estudio de la interacción mamá-bebé prematuro a través de la escala Brazelton y algunas implicaciones sobre la salud mental de las madres

Amiel-Tison C. (1999a). Neurologie Perinatale.Paris: Ed Masson.

Amiel-Tiso C. (1999b). L’enfant nouveau-né un cerveau pour la vie. Inserm.

Beck A. (1978). Beck Depression inventory. The psycological corporation, San Antonio, TX: Harcourt Brace Jovanovich.

Belsky J, \& Fearon P. (2002). Early attachment security, subsequent maternal sensitivity,and later child development: Does continuity in development depend upon continuity of caregiving? Attachment $\mathcal{E}$ Human Development, 4 (3), 361-387. doi:10.1080/14616730210167267.

Bener A. (2013). Psychological distress among postpartum mothers of preterm infants and associated factors: a neglected public health problem. Rev Bras Psiquiatr. 35(3): 231-6. http://dx.doi. org/10.1590/1516-4446-2012-0821.

Bowlby J. (1982). Caring for children: some influences on its development. In Parenthood, ed. R. S. Cohen, S. H. Weissman, and B. J. Cohler, New York: The Guilford Press.

Bowlby J. (1995). Una base Segura. Buenos Aires. Paidós.

Bowlby J. (1998). El apego. El apego y la pérdida. Barcelona: Paidós.

Brazelton, T. B. (1973a). Neonatal behavioral assessment scale. Philadelphia: Lippincott.

Brazelton, T.B. (Ed.) (1973b). Neonatal Behavioural Assessment Scale. London: MacKeith Press.

Brazelton T, B. \& Nugent J. K. (1995). Neonatal Behavioral Assessment Scale. $3^{a}$ edición. Mac Keith Press.

Brazelton, T. \& Nugent K. (1997). Escala para la evaluación del comportamiento neonatal ( $1^{a}$ edición). Barcelona. Ed Paidós.

Brazelton, T. B., Tronick, E., Adamson, L., Als, H., \& Wise, S. (1975). Early mother-infant reciprocity. In M. A. Hofer (Ed.). The parentinfant relationship (pp. 137-155). London: Ciba.

Brazelton, T.B. (1984). Neonatal Behavioral assessment scale $2^{a}$ edición. Clinics in Developmental Medicine $N^{\circ}$ 88, Londres: Ed. Spastics Internacional Medical Publications (SIMP).

Brazelton, T. (2001). Momentos clave en la vida de tu hijo. Barcelona: Ed. Plaza \& Janés. 
Charpak, N., Gabriel Ruiz, J., Zupan, J., Cattaneo, A., Figueroa, Z., Tessier, R., ... \& Mokhachane, M. (2005). Kangaroo mother care: 25 years after. Acta Paediatrica, 94(5), 514-522.

Chu, A. \& Lieberman, A. (2010). Clinical implication of traumatic stress from birth to age five. The annual review of clinical psychology. 6, (1) 469 - 494. https://doi.org/10.1146/annurev.clinpsy.121208.131204

Costa, R., Figueiredo, B., Tendais, I., Conde, A., Pacheco, A. \& Teixeira, C. (2010). Brazelton Neonatal Behavioral Assessment Scale: A psychometric study in a Portuguese sample. Infant Behavior and Development. 33, (4) 510-517, https://doi.org/10.1016/j.infbeh.2010.07.003.

Costas, C., Fornieles, A., Botet, F., Boatella, E. \& De Cáceres M. (2007). Evaluación psicométrica de la Escala de Brazelton en una muestra de recién nacidos españoles. Psicothema, 19(1), 140-149.

De cero a siempre (2013). El aumento del embarazo de adolescentes en Colombia (boletin No 2). Recuperado de https://goo.gl/DVM2VE.

De Chateau, P., \& Wiberg, B. (1977). Long term effect on mother-infant behavior of extra contact during the first hour post partum: Vol. 2. Follow-up at three months. Acta Paediatrica Scandinava,,66 (2),137- 143. DOI: 10.1111/j.1651-2227.1977.tb07825.x.

DiVitto, B., \& Goldberg, S. (1979). The effects of newborn medical status on early parent-infant interaction. In T. M. Field, A. M. Sostek, S. Goldberg, \& H. H. Sherman (Eds.), Infants bom at risk (pp. 311332). New York: Spectrum Books.

Eckerman, C. O., Hsu, H.-C., Molitor, A., Leung, E. H. L., \& Goldstein, R. F. (1999). Infant arousal in an en-face exchange with a new partner: Effects of prematurity and perinatal biological risk. Developmental Psychology, 35(1), 282-293.http://dx.doi.org/10.1037/00121649.35.1.282.

Fava G, Zorzi C, Bottos M. (1992). Los hijos de las máquinas. La vida de los niños internados en terapias intensivas neonatales. Ed. Nueva Visión. Buenos Aires.

Feldman, R, . \& Eidelman A. (2003). Direct and indirect effects of breast milk on neurobehavioral and cognitive development of pre- 
mature infants. Developmental Psychobiology, 43(2), 109-119. Doi:10.1002/dev.10126.

Feldman, R. \&. Eidelman, A. I (2006). Neonatal State Organization, Neuromaturation, Mother-Infant Interaction, and Cognitive Development in Small-for-Gestational-Age Premature Infants. Pediatrics, 118(3). Doi:10.1542/peds.2005-2040.

Feldman, R. (2007). Parent-infant synchrony and the construction of shared timing: Psychological precursors, developmental outcomes, and risk conditions. Journal of Child Psychology and Psychiatry, 48, 329-354. doi:10.1111/j.1469-7610.2006.01701.x.

Feldman, R. (2009). The development of regulatory functions from birth to 5 years: Insights from premature infants. Child Development: 80 (2), 544-561. DOI: 10.1111/j.1467-8624.2009.01278.x.

Field T. (1977). Effects of early separation, interactive deficits, and experimental manipulations on infant-mother face-to-face interaction. Child Development. 48 (3):763-771. http://dx.doi. org/10.2307/1128325.

Field, T. (1995). Massage therapy for infants and children. Journal of Behavior and Developmental Pediatrics, 16, 105-111. http://dx.doi. org/10.1097/00004703-199504000-00008.

Forcada-Guex, M., Pierrehumbert, B., Borghini, A., Moessinger, A., \& Muller-Nix, C. (2010). Early dyadic patterns of mother-infant interactions and outcomes of prematurity at 18 months. Pediatrics: 118 (1), 107-114. DOI: 10.1542/peds.2005-1145.

Fuertes, M., Lopes-Dos-Santos P., Beehgly, M.\& Tronick E. (2009). Infant coping and maternal interactive behavior predict attachment in a Portuguese sample of healthy preterm Infants. European Psychologist, 14(4): 320-331. http://dx.doi.org/10.1027/10169040.14.4.320.

González S. F. (2009). Nacer de nuevo: la crianza de los niños prematuros: La relación temprana y el apego. Cuadernos de Psiquiatría y Psicoterapia del niño y del adolescente, 48 (1) 61 - 80. http:// www.sepypna.com/documentos/articulos/crianza-ninos-prematuros-gonzalez-serrano.pdf. 
González S. F. (2010). Nacer de nuevo: la crianza de los niños prematuros: aspectos evolutivos. Atención y acompañamiento al bebé y la familia. Cuadernos de Psiquiatría y Psicoterapia del niño y del adolescente. 49 (1) 133-152. Recuperado de http://www.sepypna.com/ documentos/articulos/nacer-crianza-ninos-prematuros.pdf.

Green, J., Bax, M., \& Tsitsikas, H. (1989). Neonatal behavior and early temperament: A longitudinal study of the first six months of life. American Journal of Orthopsychiatry, 59(1), 82-93. http://dx.doi. org/10.1111/j.1939-0025.1989.tb01637.x.

Gunnar M, \& Quevedo K. (2007). The neurobiology of stress and development. Annual Review of Psychology, 58 (1), 145-73. https://doi. org/10.1146/annurev.psych.58.110405.085605.

Habersaat, S., Pierrehumbert, B., Forcada-Guex, M., Nessi, J., Ansermet, F., Müller-Nix, C., \& Borghini, A. (2014). Early stress exposure and later cortisol regulation: Impact of early intervention on mother-infant relationship in preterm infants. Psychological Trauma: Theory, Research, Practice, and Policy, 6(5), 457-464. http://dx.doi.org.ezproxy.unal.edu.co/10.1037/a0033878.

Hawthorne J. (2005). Using the Neonatal Behavioural Assessment Scale to support parent-infant relationships. Infant, 1(6): 213-218. http:// www.infantjournal.co.uk/pdf/inf_006_irs.pdf.

Hayes, B., \& Sharif, F. (2009). Behavioural and emotional outcome of very low birth weight infants-Literature review [Review]. Journal of Maternal-Fetal and Neonatal Medicine, 22, (1) 849-856. doi:10.1080/ 14767050902994507.

Holditch-Davis, D., White-Traut, R. C., Levy, J. A., O'Shea, T.M., Geraldo, V. \& David, R. J. (2014). Maternally administered interventions for preterm infants in the NICU: Effects on maternal psychological distress and mother-infant relationship. Infant Behavior and Development,37 (4), 695-710. https://doi.org/10.1016/j.infbeh.2014.08.005.

Holmes, D. L., Nagy, J. N., Slaymaker, F., Sosnowski, R. J., Prinz, S. M., $\&$ Pasternak, J. F. (1982). Early influences of prematurity, illness, and prolonged hospitalization on infant behavior. Developmental Psychology, 18(5), 744-750. http://dx.doi.org/10.1037/00121649.18.5.744. 
Honglin Zhu, Kek Khee Loo, Lihua Min, Qiongying Yin, Hong Luo, Lian Chen \& Shohei Ohgi. (2007). Relationship between neurobehaviours of Chinese neonates and early mother-infant interaction. Journal of Reproductive and Infant Psychology, 25:2, 106-121, DOI:10.1080/02646830701292340.

Institute of Medicine US. (2007). Committee on Understanding Premature Birth and Assuring Healthy Outcomes; Behrman RE, Butler AS, editors. Preterm Birth: Causes, Consequences, and Prevention. Washington (DC): National Academies Press (US).

Jacobson S., Fein G. G, Jacobson J. L, Schwartz, P. M \& Dowler, J. K. (1984). Neonatal correlates of prenatal exposure to smoking, caffeine, and alcohol. Infant and Child Development, 7(3), 253-265. https://doi. org/10.1016/S0163-6383(84)80041-7.

Jacobson, J. L., Fein, G. G., Jacobson, S. W., \& Schwartz, P. M. (1984). Factors and clusters for the brazelton scale: An investigation of the dimensions of neonatal behavior. Developmental Psychology, 20(3), 339-353. http://dx.doi.org/10.1037/0012-1649.20.3.339.

James-Roberts, \& Halil, T. (1991). Infant crying patterns in the first year: Normal community and clinical findings. Journal of Child Psychology and Psychiatry, 32. 951-968. Doi:10.1111/j.1469-7610.1991. tb01922.x.

James-Roberts, Wolke D. (1988). Convergence and discrepancies among mother' and professionals' assessments of difficult neonatal behavior. Journal of Child Psychology and Psychiatry, 29: 21-42. Doi:10.1111/j.1469-7610.1988.tb00686.x 29: 21-42.

Klein, P. \& Feldman R. (2007). Mothers' and caregivers' interactive and teaching behaviour with toddlers. Early Child Development and Care, 177 (4): 383-402. https://doi.org/10.1080/03004430600551682.

Korja, R., Maunu, J., Kirjavainen, J., Savonlahti, E., Haataja, L., Lapinleimu, H., ... \& PIPARI Study Group. (2008). Mother-infant interaction is influenced by the amount of holding in preterm infants. Early human development, 84(4), 257-267.

Korner A \& Thom V. (1990). Neurobehavioral Assessment of the Preterm Infant. Nueva York: The Psychological Corporation. Harcourt, Brace \& Jovanovich, Inc. 
Lehmann, J., Stohr, T., \& Feldon, J. (2000). Long-term effects of prenatal stress experiences and postnatal separation on emotionality and attentional processes. Behavioral Brain Research, 107(2), 133-144. https://doi.org/10.1016/S0166-4328(99)00122-9.

Leonardelli, E. (2009). Desarrollo de un modelo para la observación sistemática de situaciones interactivas lúdicas madre-niño. I congreso internacional de investigación y práctica profesional en psicología, XVI jornadas de investigación, V Encuentro de investigadores en psicología del MERCOSUR, Tomo II. Ediciones de la Facultad de Psicología, Universidad de Buenos Aires. Argentina.

Lester B, Hoffman Joel, \& Brazelton T. (1985). The rhythmic structure of mother-infant interaction in term and preterm infants. Child Development, 56(1), 15-27. DOI: 10.2307/1130169.

Macey, T. J., Harmon, R. J., \& Easterbrooks, M. A. (1987). Impact of premature birth on the development of the infant in the family. Journal of Consulting and Clinical Psychology, 55(6), 846-852. http://dx. doi.org./10.1037/0022-006X.55.6.846.

Marrone M. (2001). La Teoría Del Apego: Un enfoque actual. Madrid: Editorial Psimática.

McDonough, S. (2005). Interaction guidance: Promoting and nurturing the caregiving relationship. In A. J. Sameroff, S. C. McDonough, \& K. L. Rosenblum (Eds.), Treating parent-infant relationship problems: Strategies for intervention (pp. 79-86). New York, NY: Guilford Press.

Meier P, Wolke D, Gutbrod T, Rust, L. (2003). The influence of infant irritability on maternal sensitivity in a sample of very premature infants. Infant and Chile Development, 12(2): 159-166. Doi:10.1002/ icd.284.

Myers B, Jarvis P, Creasey G, Kerkering K. (1992). Prematurity and respiratory illness: Brazelton scale (NBAS) performance of preterm infants with bronchopulmonary dysplasia (BPD), respiratory distress syndrome (RDS), or no respiratory illness. Infant Behavior \& Development, 15(1): 27-41. https://doi.org/10.1016/01636383(92)90004-P.

Neu, M., \& Robinson, J. (2010). Maternal holding of preterm infants during the early weeks after birth and dyad interaction at six months. Jour- 
Estudio de la interacción mamá-bebé prematuro a través de la escala Brazelton y algunas implicaciones sobre la salud mental de las madres

nal of Obstetric, Gynecologic, and Neonatal Nursing, 39, (4), 401414. https://doi.org/10.1111/j.1552-6909.2010.01152.x.

Nicolaou M, Rosewella R, Marlow N, \& Glazebrook G. (2009). Mothers' experiences of interacting with their premature infants. Journal of Reproductive and Infant Psychology, 27(2): 182-194. https://doi. org/10.1080/02646830801922796.

Opinión \& Salud (2015). De cada 100 niños que nacen en Colombia, 12 son prematuros. Revista digital Recuperado de http://bit.ly/2EAC3f9.

Papalia, D. \& Wendkos, S. (1992) Psicología del Desarrollo: de la infancia a la adolescencia (5ta edición). México: Editorial McGraw-Hill.

Pérez-López J. (2006). Modelos explicativos del desarrollo aplicados a la Atención Temprana. En J. Pérez-López \& A. Brito de la Nuez (Coords.), Manual de Atención Temprana; (pp. 27-42). Madrid: Editorial Pirámide.

Phillips R, Sharma R, Premachandra B, Vaughn A, Reyes-Lee M. (1996). Intrauterine exposure to cocaine: Effect on neurobehavior of neonates. Infant Behavior \& Development. 19(1); 71-81. https://doi. org/10.1016/S0163-6383(96)90045-4.

Pignotti, M. S., \& Donzelli, G. (2008). Perinatal care at the threshold of viability: An international comparison of practical guidelines for the treatment of extremely preterm births. Pediatrics, 121(1), 193-198. doi:10.1542/peds.2007-0513.

Puig-Calsina S, Obregón-Gutiérrez N, Calle del Fresno S, Escalé-Besa M, Cantó-Codina L, Goberna- Tricas J. (2018). Ansiedad y depresión en las madres de recién nacidos prematuros. Estrategias de intervención y revisión de la bibliografía. Matronas Prof. 19(1): 21-27. Recuperado de http://www.federacion-matronas.org/wp-content/ uploads/2018/04/Revision_Ansiedad-1.pdf.

RAE- Real Academia Española (2018). Diccionario de la lengua española (23.a ed.) Madrid, España. Recuperado de http://dle.rae.es/?id=LsCpk2t.

Redacción el país (2014). En Colombia los partos prematuros representan el $12 \%$ de los nacimientos. Recuperado de https://goo.gl/iLTvQ3.

Reed, D. M., \& Stanley, F. J. (1977). The epidemiology of prematurity. Baltimore, Md.: Urban \& Schwansenberg, USA. 
Richardson, G., Hamel, S., Golschmindt, L. \& Day, N. (1996). The effects of prenatal cocaine use on neonatal neurobehavioral status. Neurotoxicology and teratology. 18 (5), 519-528. https://doi. org/10.1016/0892-0362(96)00062-1.

Romero-Acosta K., \& Ruiz, F. (2015). El trastorno de estrés postraumático en niños preescolares: una revisión literaria. Katharsis, 20 (1), 145 - 167. Recuperado de http://revistas.iue.edu.co/index.php/katharsis/article/view/732/1036.

Ruiz, A.L. (2004). Un nacimiento antes de tiempo. Subjetividad y Procesos Cognitivos; 256-280. Recuperado de: http://dspace.uces.edu. ar:8180/xmlui/bitstream/handle/123456789/258/Un_nacimiento_ antes_de_tiempo.pdf?sequence $=1$.

Scafidi, F. A., Field, T. M., Schanberg, S. M., Bauer, C. R., Tucci, K., Roberts, J., Morrow, C., \& Kuhn, C. (1990). Massage stimulates growth in preterm infants: A replication. Infant Behavior and Development, 13 (2),167-188. https://doi.org/10.1016/0163-6383(90)90029-8.

Shin, H., Park, Y., Ryu, H. \& Seomun G. (2008). Maternal sensitivity: A concept analysis. Journal of Advanced Nursuring, 6 (3): 304-314. DOI: 10.1111/j.1365-2648.2008.04814.x.

Smeriglio, V. L. (1981). Measures of effects of mother-newborn contact: Similarities, differences, conceptual basis and validity. In V. L. Smeriglio (Ed.), Newborns and parents: Parent-infant contact and newborn sensory stimulation. Hillsdale, N.J.: Erlbaum.

Sostek, A. M, \& Anders T. F. (1977). Relationships among the Brazelton Neonatal Scale, Bayley Infant Scales, and early temperament. Child Development;48(1), 320-323. http://dx.doi.org/10.2307/1128921.

Treyvaud, K., Anderson, V. A., Lee, K. J., Woodward, L. J., Newnham, C., Inder, T. E., ... \& Anderson, P. J. (2009). Parental mental health and early social-emotional development of children born very preterm. Journal of Pediatric Psychology, 35(7), 768-777.

Tu, M. T., Grunau, R. E., Petrie-Thomas, J., Haley, D. W., Weinberg, J., \& Whitfield, M. F. (2007). Maternal stress and behavior modulate relationships between neonatal stress, attention, and basal cortisol at 8 months in preterm infants. Developmental Psychobiology, 49(2), 150-164. doi: 10.1002/dev.20204. 
Van Zeijl, J., Mesman, J., Van Izendoorm, H.M., Bakermans-Kranenburg, J.M., Juffer, M., Stolk, N.M., Koot, M. H. \& Alink, L.R. (2006). Attachment-Based Intervention for enhancing Sensitive Discipline in Mothers of 1- to 3-Year-Old Children at Risk for Externalizing Behavior Problems: A Randomized Controlled Trial. Journal of Consulting and Clinical Psychology, 74 (6), 994-1005. http://dx.doi. org/10.1037/0022-006X.74.6.994.

Warren, S. L., Gunnar, M. R., Kagan, J., Anders, T. F., Simmens, S. J., Rones, M. \& Sroufe, L. A. (2003). Maternal panic disorder: Infant temperament, neurophysiology and parenting behaviors. Journal of the American Academy of Child \& Adolescent Psychiatry, 42,(7),814825. doi:10.1097/01.CHI.0000046872.56865.02.

White-Traut, R. C., Schwertz, D., McFarlin, B., \& Kogan, J. (2009). Salivary cortisol and behavioral state responses of healthy newborn infants to tactile-only andmultisensory interventions. Journal of Obstretric, Gynecologic, \& Neonatal Nursing, 38., (1), 22-34. http://dx.doi. org/10.1111/j.1552-6909.2008.00307.x.

Widmayer, S.M., Field, T.M (1981). Effects of Brazelton demonstrations for mothers on the development of preterm infants. Pediatrics, 67 (5), 711-714. Retrieved from https://www.ncbi.nlm.nih.gov/ pubmed/7255001.

Wiggera, A., \& Neumanna, I. D. (1999). Periodic maternal deprivation induces gender-dependent alterations in behavioral and neuroendocrine responses to emotional stress in adult rats. (2), 66, 293-302. https://doi.org/10.1016/S0031-9384(98)00300-X.

Worobey, J., \& Belsky, J. (1982). Employing the brazelton scale to influence mothering: An experimental comparison of three strategies. Developmental Psychology, 18(5), 736-743. http://dx.doi. org/10.1037/0012-1649.18.5.736.

Zeanah, C. H., Boris, N. W. \& Larrieu, J. A. (1997). Infant Development and Developmental Risk: A Review of the Past 10 Years. Journal of the American Academy of Child \& Adolescent Psychiatry, 36, (2), 165-178. https://doi.org/10.1097/00004583-199702000-00007. 